\title{
Variant Origins of Arteries in the Carotid Triangle - A Case Report
}

\author{
B. V. Murlimanju, MD; Latha V. Prabhu, MS; Mangala M. Pai, MD; \\ Dhanya Jayaprakash, MBBS; Vasudha V. Saralaya, MS
}

The left superior laryngeal artery was observed arising from the external carotid artery instead of the superior thyroid artery in the cadaver of an approximately 70 year-old Asian man. In addition, on the same side, the superior thyroid artery arose from the common carotid artery $2 \mathrm{~cm}$ before the bifurcation instead of its usual origin from the external carotid artery. From the external carotid artery, the lingual and facial arteries arose from the common linguofacial trunk. The nerves in the carotid triangle were normal in course. No variations were observed on the right side carotid system. The multiple variations in this case have not been previously described. The embryogenesis of this combination of variations is not clear, but the anatomic consequences may have important clinical implications. As angiography has gained popularity in diagnostic approaches in recent years, it is essential to be aware of these variations so that they are not overlooked in differential diagnoses. (Chang Gung Med J 2012;35:281-4)

Key words: artery, superior laryngeal, superior thyroid, common carotid, external carotid, variant origin

\begin{abstract}
A natomical variations in the carotid triangle in the neck are important, especially during surgical and radiological intervention in the region. Normally, the superior laryngeal artery (SLA) is a branch of the superior thyroid artery (STA). It runs horizontally in the carotid triangle along with the internal laryngeal nerve and pierces the thyrohyoid membrane. The artery supplies the lower part of the pharynx and upper part of the larynx. Variations in the SLA are very rare. In this case we report a variant origin of the left SLA from the external carotid artery (ECA). The common carotid artery (CCA) normally does not give off any other branches in the neck except for terminal branches, and the external and internal carotid arteries. A rare variant origin of
\end{abstract}

the STA from the left CCA is reported here. In the literature, a few variations in origin have been reported for both arteries, ${ }^{(1-3)}$ but the combination of variations reported in this case has not been previously described, to the best of our knowledge. Understanding the surgical anatomy of the STA is necessary for successful radical neck dissection and to minimize postoperative complications in a bloodless surgical field. Also, knowledge of variations in the origins of these arteries is important for surgical procedures in the neck, such as emergency cricothyroidotomy, radical neck dissection, catheterization, reconstruction of aneurysms and carotid endarterectomy. The variations in the present case are reported and compared with those reported before.

From the Department of Anatomy, Centre for Basic Sciences, Kasturba Medical College, Manipal University, Bejai, Mangalore, Karnataka, India.

Received: Nov. 16, 2011; Accepted: Jan. 30, 2012

Correspondence to: Dr. B. V. Murlimanju, Department of Anatomy, Kasturba Medical College, Manipal University, Bejai, Mangalore, India. Department of Anotomy, Kasturba Medical College, Bejai, Mangalore - 575004, India. Tel: 91-824-2211746; Fax: 91-824-2421283; E-mail: flutemist@ gmail.com 


\section{CASE REPORT}

During an anatomy dissection class for undergraduate dental students, we found three variations in the left carotid triangle in the cadaver of an approximately 70 year-old Asian man. The CCA divided into the external and internal carotid arteries at the level of the upper border of the thyroid cartilage as reported in textbooks. However the SLA arose from the ECA and the STA arose from the CCA, $2 \mathrm{~cm}$ before the bifurcation (Figure). The SLA arose $6 \mathrm{~mm}$ distal to the bifurcation of the CCA. The lingual and facial arteries originated from the linguofacial trunk which arose from the ECA. The remaining branches of the left ECA were normal in origin. The arterial system was carefully dissected and photographed (Figure). The CCA was $9.8 \mathrm{~cm}$ long. The nerves of the carotid triangle were normal in course. The branching pattern on the right side carotid system was normal without any variations.

\section{DISCUSSION}

The STA is frequently used as a recipient vessel for microvascular free tissue transfer in head and neck surgery, for selective embolization of thyroid and head and neck tumors, and as a landmark for identifying the external branch of the superior laryngeal nerve in thyroid surgery. ${ }^{(4)}$ Consequently, it is important to have a clear understanding of variations in the anatomy of this artery and to be able to distinguish it from one of its key branches in situations in which normal anatomical variation could lead to them being confused. ${ }^{(4)}$ Lo et al. reported that the origin of STA appeared to be related to the level of the CCA bifurcation. ${ }^{(5)}$ When the CCA had a relatively low bifurcation, such as at the lamina of the thyroid cartilage, the STA tended to originate from the ECA. In contrast, when the CCA had a high bifurcation, the STA tended to originate from the CCA. In the present case, the CCA was $9.8 \mathrm{~cm}$ long. But, the mean length of the left CCA has been reported to be $12.1 \pm 0.2 \mathrm{~cm} .{ }^{(6)}$ This suggests that the CCA in our case was short, which means there was a low bifurcation. This finding is not consistent with the observations of Lo et al., as the STA arose from CCA which had a low bifurcation. ${ }^{(5)}$

In rare cases, the SLA might arise from the lingual, facial or ascending pharyngeal arteries. ${ }^{(7)}$ The
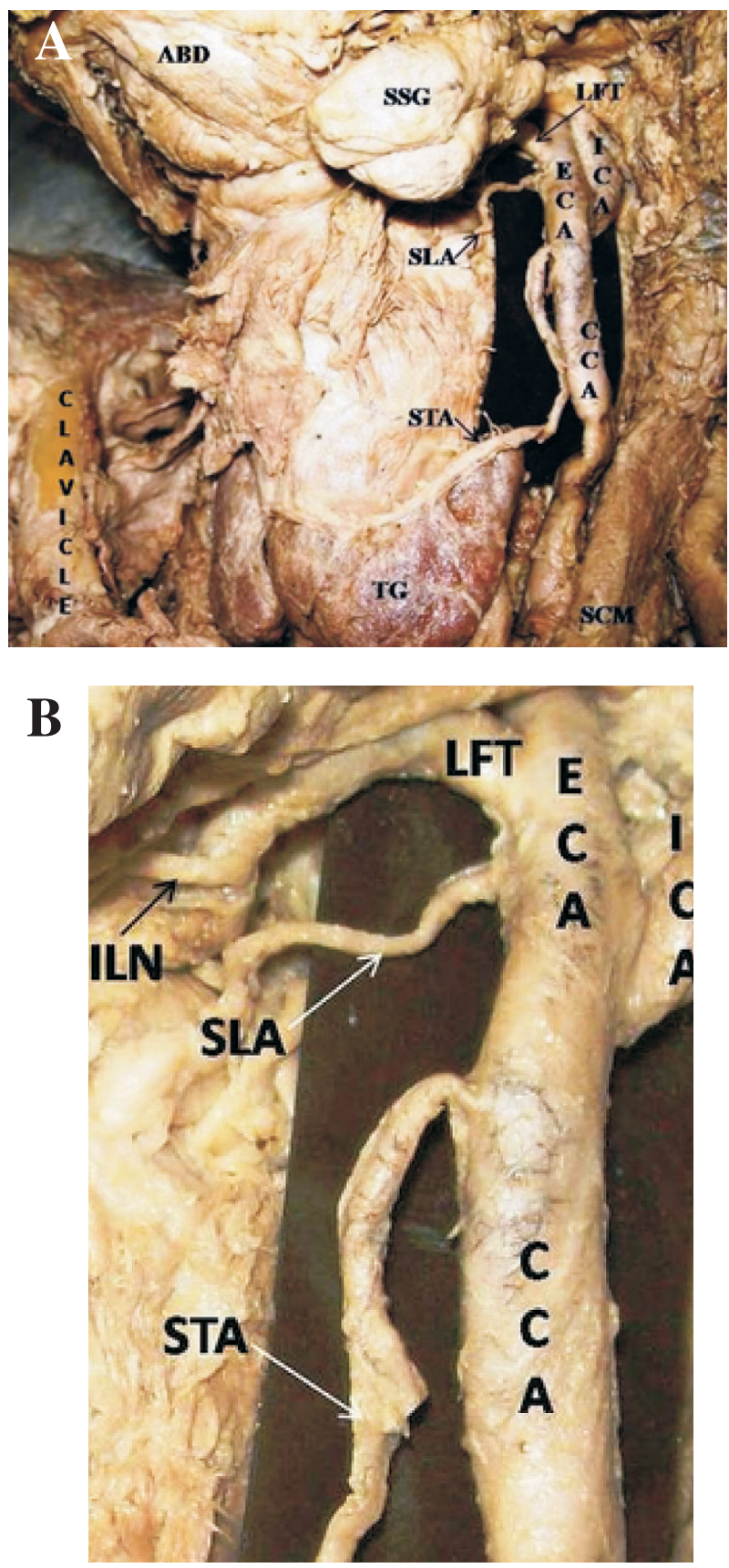

Figure (A) Photograph showing variant origins of the superior laryngeal and superior thyroid arteries in the cadaver of an Asian man. (B) Close up view of the same variation. Abbreviations used: TG: thyroid gland; SSG: submandibular salivary gland; SCM: sternocleidomastoid muscle; CCA: common carotid artery; ECA: external carotid artery; STA: superior thyroid artery; SLA: superior laryngeal artery; LFT: linguofacial trunk; ICA: internal carotid artery; ABD: anterior belly of digastric; ILN: internal laryngeal nerve. 
distance of origin of the SLA from the carotid bifurcation in cases in which the artery arises from ECA has been reported by Vazquez et al. ${ }^{(4)}$ In their study, the mean distance of the SLA origin to the carotid bifurcation was $0.5 \mathrm{~cm}$. They also reported that the SLA arose in an area similar to that which might be expected for the origin of the STA. In the present case, the distance from the bifurcation was $0.6 \mathrm{~cm}$, which is similar to their findings.

The STA is normally considered to be the first branch arising from the ECA and is used as a surgical landmark for differentiating the external from the internal carotid artery, since no cervical branches normally arise from the latter or from the carotid bifurcation. In this case, the first artery arising from ECA was the SLA rather than the STA, so this variation must be kept in mind. The accepted site of ECA ligation is inferior to the origin of the STA. However, its closeness to the carotid bifurcation might cause difficulty in surgery, and it is accepted that ligation above the STA may preserve good collateral circulation. ${ }^{(4)}$ Because of the variable origin of the STA and the fact that the SLA usually arises less than $1 \mathrm{~cm}$ from the origin of the ECA, the STA is not a reliable landmark for identification of the ECA or identification of a safe point for its ligation. The only reliable method to identify the ECA is to identify several collateral branches of the ECA before ligation..$^{(4)}$ It was reported that these variations can be critical, for example in a tracheotomy, in surgery on the thyroid and parathyroid glands and in tumor resection in the neck. ${ }^{(8)}$

The development of the ECA and its branches is incompletely understood. ${ }^{(9)}$ The variations might be related to changes in the transformation of the aortic arch system in the embryo. ${ }^{(10)}$ The ECA is formed after a complicated process of angiogenesis. ${ }^{(11)}$ It includes annexation and regression of the vessels with some remodeling. The development begins through a combination of outgrowths from some vessels, involution of others and assimilation of preexisting channels that arise from undifferentiated precursor vessels. ${ }^{(9)}$ The development of the hyostapedial artery which links the neural crest arterial system and the ventral pharyngeal artery is an important event. ${ }^{(12)}$ The carotid bifurcation area and the CCA derive from the third aortic arch, while the ECA derives from the first arch with some contribution from the second aortic arch. ${ }^{(13)}$ According to
Larsen, ${ }^{(12)}$ the trunks of the ECA arise as de novo outgrowths from the third aortic arches. Various ventral vestiges of the first and second aortic arch arteries and ventral aorta form the ventral pharyngeal artery which later becomes the main stem of the ECA. The ventral pharyngeal artery undergoes further modifications and gives the identity of the ECA and its branches. Deviation in the normal stages of annexation and regression during angiogenesis leads to the anomalous structures. ${ }^{(11)}$ The embryological basis of the variations observed in the present case is unclear. They could occur because of persistence of channels which normally disappear or from disappearance of vessels which normally persist.

Although the linguofacial trunk and origin of the STA from the CCA are common, an SLA arising from the ECA is very rare. ${ }^{(14)}$ Hollinshead's textbook reports that the STA originates from the CCA in 16\% of cases. ${ }^{(15)}$ Variations in the origin of the STA and SLA from the carotid arterial tree mean that there is a significant possibility of their misidentification during surgery. These variations are important for general surgeons, neurosurgeons, ear, nose and throat surgeons and radiologists. Severe complications could occur when radiographic evaluation or surgical proceedings are done in the neck without prior knowledge of variations. Since angiographic studies have gained importance in diagnostic approaches in recent years, and also because these variations are important in surgical approaches, this case was reported in detail and compared with similar instances in the literature.

\section{REFERENCES}

1. Anu VR, Pai MM, Rajalakshmi R, Latha VP, Rajanigandha V, D'Costa S. Clinically-relevant variations of the carotid arterial system. Singapore Med J 2007;48:566-9.

2. Ozgur Z, Govsa F, Celik S, Ozgur T. Clinically relevant variations of the superior thyroid artery: an anatomic guide for surgical neck dissection. Surg Radiol Anat 2009;31:151-9.

3. Rusu MC, Nimigean V, Banu MA, Cergan R, Niculescu $\mathrm{V}$. The morphology and topography of the superior laryngeal artery. Surg Radiol Anat 2007;29:653-60.

4. Vazquez T, Cobiella R, Maranillo E, Valderrama FJ, McHanwell S, Parkin I, Sanudo JR. Anatomical variations of the superior thyroid and superior laryngeal arteries. Head Neck 2009;31:1078-85. 
5. Lo A, Oehley M, Bartlett A, Adams D, Blyth P, Al-Ali S. Anatomical variations of the common carotid artery bifurcation. ANZ J Surg 2006;76:970-2.

6. Ribeiro RA, Ribeiro JAS, Rodrigues Filho OA, Caetano GA, Fazan VPS. Common carotid artery bifurcation levels related to clinical relevant anatomical landmarks. Int J Morphol 2006;24:413-6.

7. Nayak SB, Soumya KV. Neurovascular variations in the carotid triangle. International Journal of Anatomical Variations 2008;1:17-8.

8. Iterezote AM, Medeiros AD, Barbosa Filho RCC, Petrella $S$, Andrade Junior LC, Marques SR, Prates JC. Anatomical variation of the brachiocephalic trunk and common carotid artery in neck dissection. Int $\mathbf{J}$ Morphol 2009;27:601-3.

9. Osborn AG, Jacobs JM. External carotid artery. In: Osborn AG, ed. Diagnostic Cerebral Angiography. 2nd ed. Philadelphia: Lippincott Williams and Wilkins, 1999:31-56.

10. Eid N, Ito Y, Otsuki Y. Anomalous branching pattern of external carotid artery: Clinical relevance to cervicofacial surgery. Clin Anat 2011;24:953-5.

11. Mahendrakar MA. Variation in the branching pattern of external carotid artery; a case report. J Anat Soc India 2007;56:47-51.

12. Larsen WJ. Development of the vasculature. In: Human Embryology. 2nd ed. New York: Churchill Livingstone, 1997:191-5.

13. Natsis K, Raikos A, Foundos I, Noussios G, Lazaridis N, Njau SN. Superior thyroid artery origin in Caucasian Greeks: A new classification proposal and review of the literature. Clin Anat 2011;24:699-705.

14. Standring S, Ellis H, Healy JC, Johnson D, William A, Collins P. Gray's Anatomy: The anatomical basis of clinical practice. 39th ed. London: Elsevier, Churchill Livingstone, 2005:543-7.

15. Hollinshead WH. Anatomy for Surgeons. Vol 1. Head and Neck, Philadelphia: Haeber Harper International, 1966;564-76. 